\title{
Primary thyroid lymphoma versus Riedel's thyroiditis.
}

Anna Kolodziejczyk ${ }^{1}$, Katerina Karamali ${ }^{2}$, Dae Kim ${ }^{1,2}$

1. St George's, University of London

2. St George's University Hospital Trust
St George's

University of London

\section{BACKGROUND}

- Primary thyroid lymphoma (PTL) and Riedel's thyroiditis (RT) are rare and diagnostically challenging entities ${ }^{1,2}$.

- Both can present as a rapidly enlarging thyroid mass and airway compromise due to compression of surrounding anatomical structures ${ }^{1,2}$.

- RT involves the overgrowth of progressively fibrosing connective tissue, often seen on diagnostic open biopsy.2.

- It has been successfully treated with corticosteroids, tamoxifen and rituximab ${ }^{3}$, however, debulking surgery is indicated in the presence of compression symptoms ${ }^{2}$.

- PTL is usually diagnosed on core biopsy, however certain immunobiological markers can aid diagnosis. PTL is treated with chemotherapy and debulking surgery is not advised ${ }^{1}$.

\begin{tabular}{l} 
CASE SUMMARY \\
\hline - A 59 year old lady presented with a rapidly enlarging thyroid \\
mass and voice hoarseness (Fig.1). \\
Initial core biopsy and open thyroid biopsy showed severe \\
inflammation consistent with RT. \\
- Initial corticosteroid and tamoxifen therapy resulted in \\
significant improvement. \\
- Within weeks, despite addition of Rituximab, an emergency \\
tracheostomy was required due to disease progression \\
causing bilateral vocal cord palsy and dysphagia. \\
Further core biopsies then revealed a diffuse large B-cell \\
lymphoma and remission was observed with R-CHOP \\
therapy.
\end{tabular}

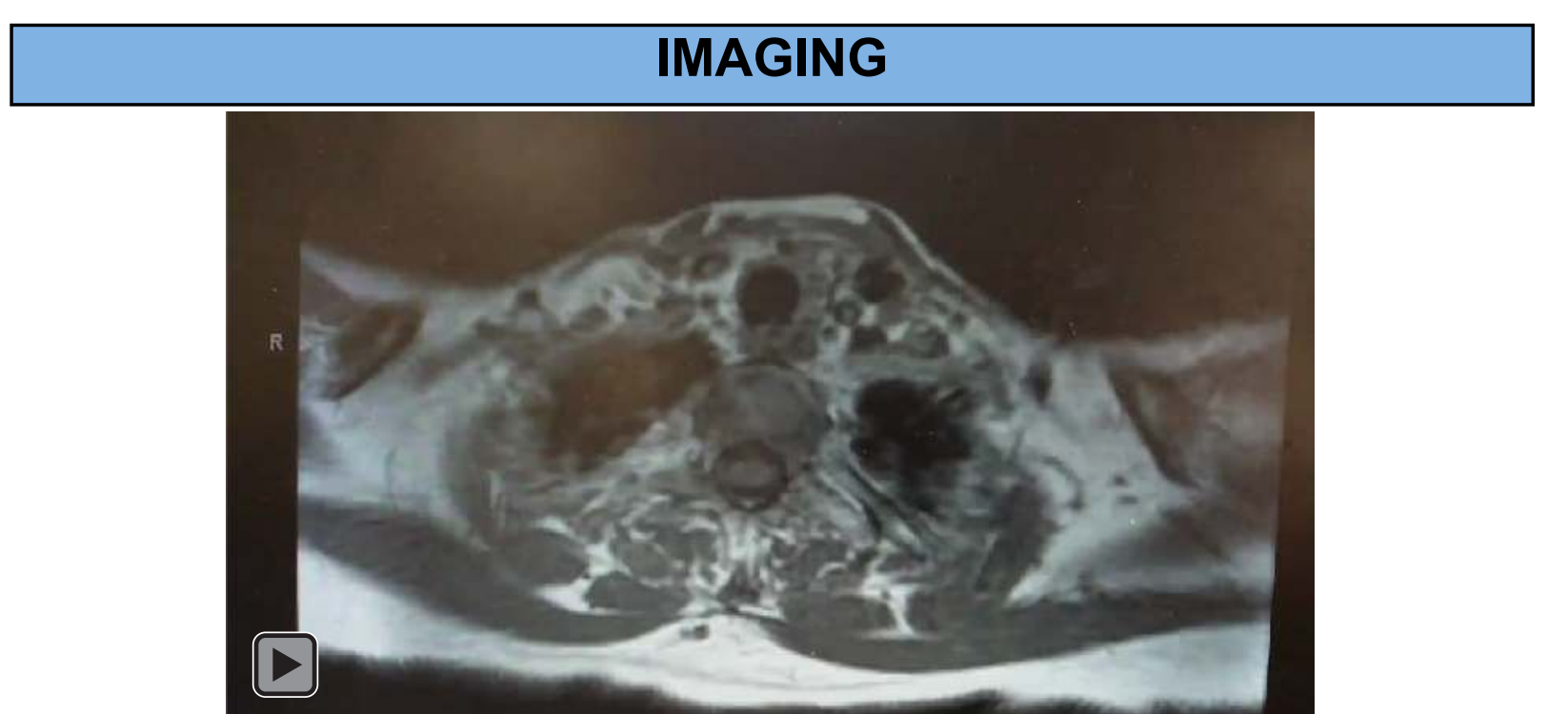

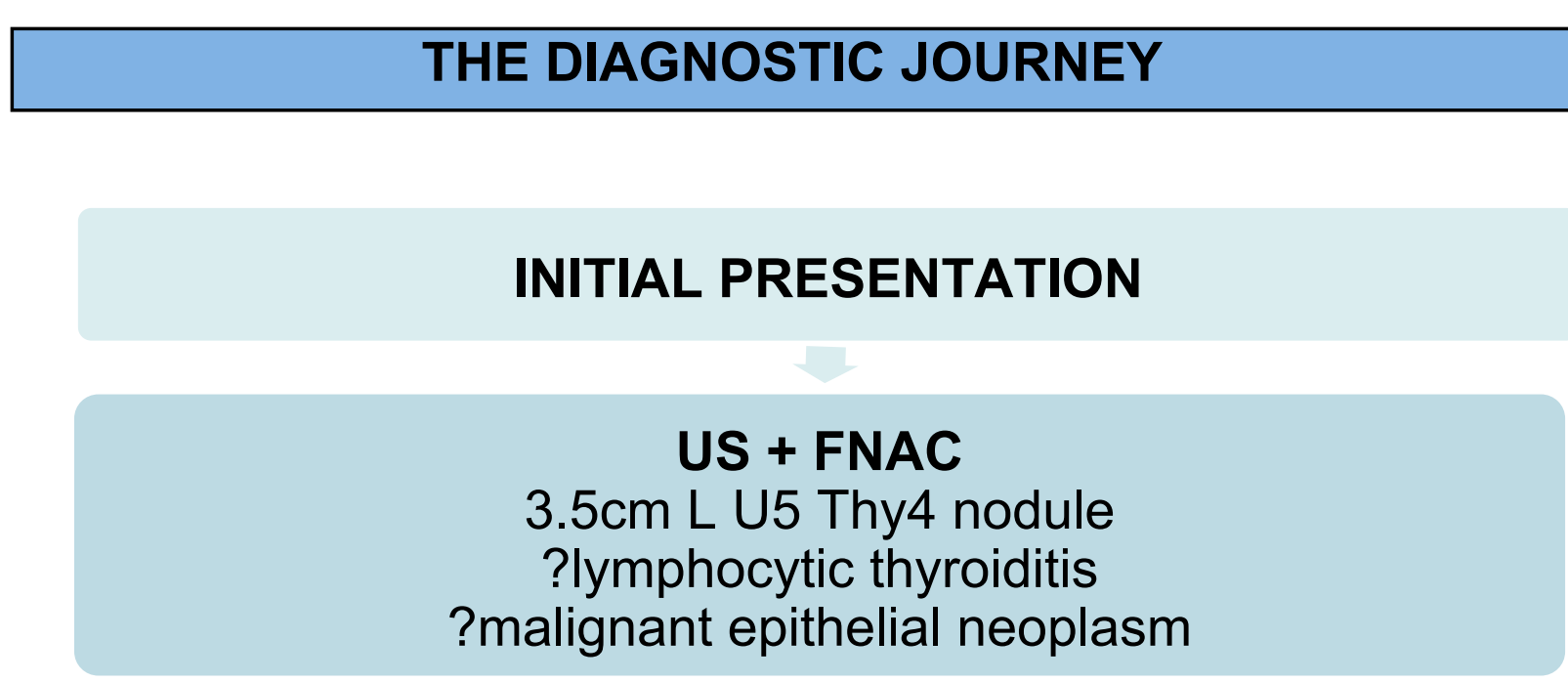
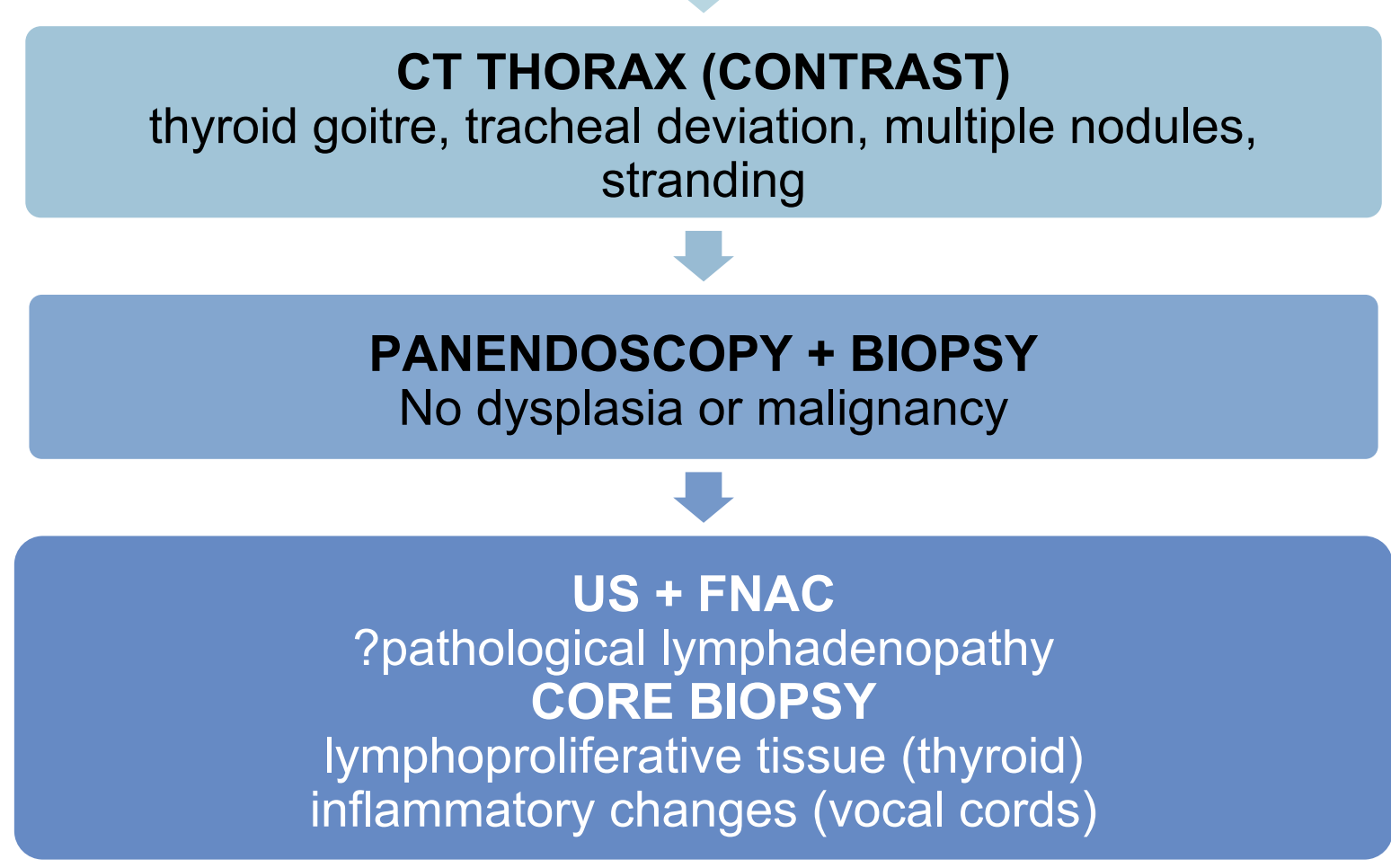

$\checkmark$

EXTERNAL OPEN BIOPSY

inflammatory infiltrate and fibrosis

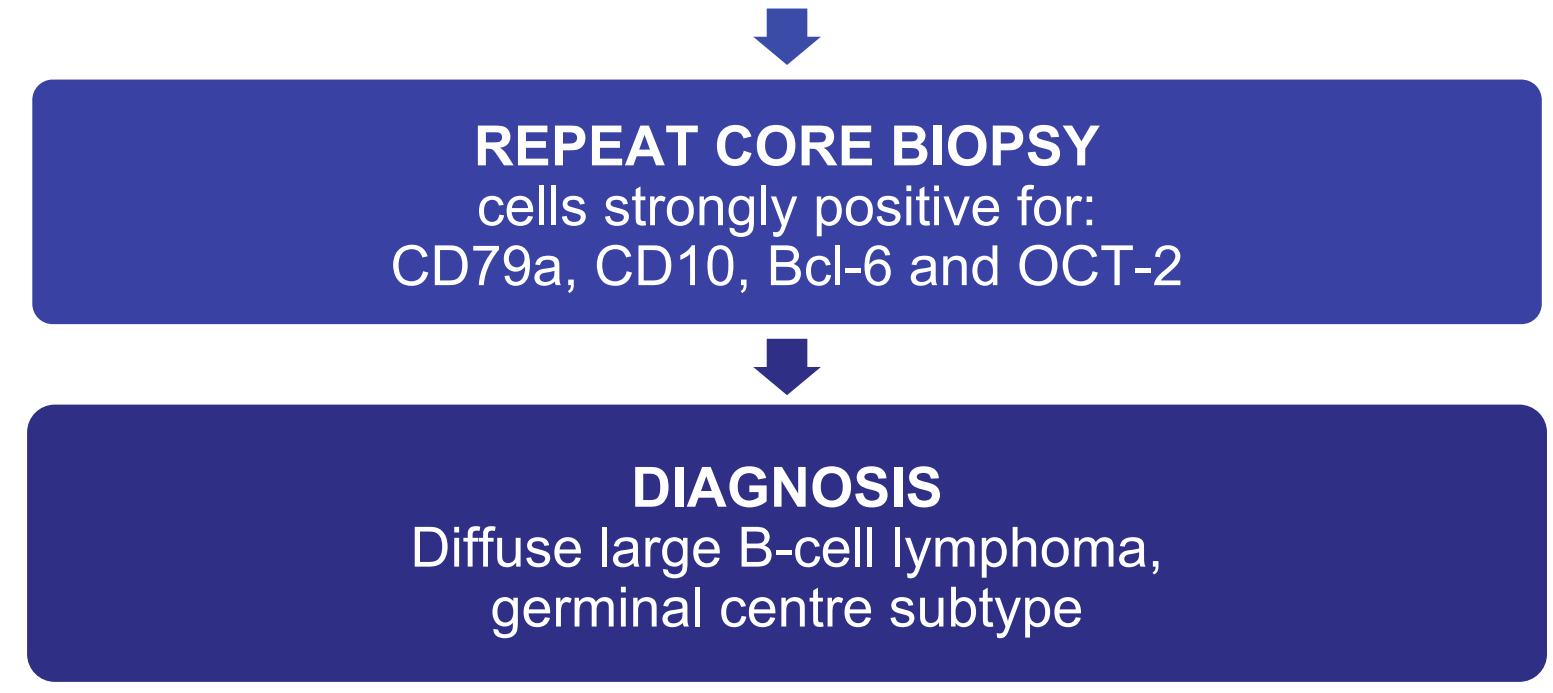

Figure 1: cervical MRI scan performed before R-CHOP treatment

\begin{tabular}{|l|}
\hline \multicolumn{1}{|c|}{ DISCUSSION } \\
\hline - Similar cases of lymphoma resembling RT and vice versa have been previously reported ${ }^{4,5}$. \\
- The delay in diagnosis resulted in a requirement for difficult airway management. \\
- It was safer for the tracheostomy to be performed by a Head \& Neck consultant surgeon, as the regional anatomy was distorted. \\
Failure to perform the surgery successfully on first attempt could have lead to fatal complications.
\end{tabular}

\section{CONCLUSIONS}

Rarely, RT can present as a rapidly enlarging neck mass, however, in such aggressive cases, a high index of clinical suspicion and repeat biopsies should be considered to exclude lymphoma.

- The diagnostic approach could potentially be improved by supplementing core biopsy with additional haematological investigations.

- A timely diagnosis is crucial due to rapid disease progression and potential significant morbidity resulting from surgery.

- Early involvement of the Haematology team is key because, even in cases of RT, chemotherapy can control disease progression.

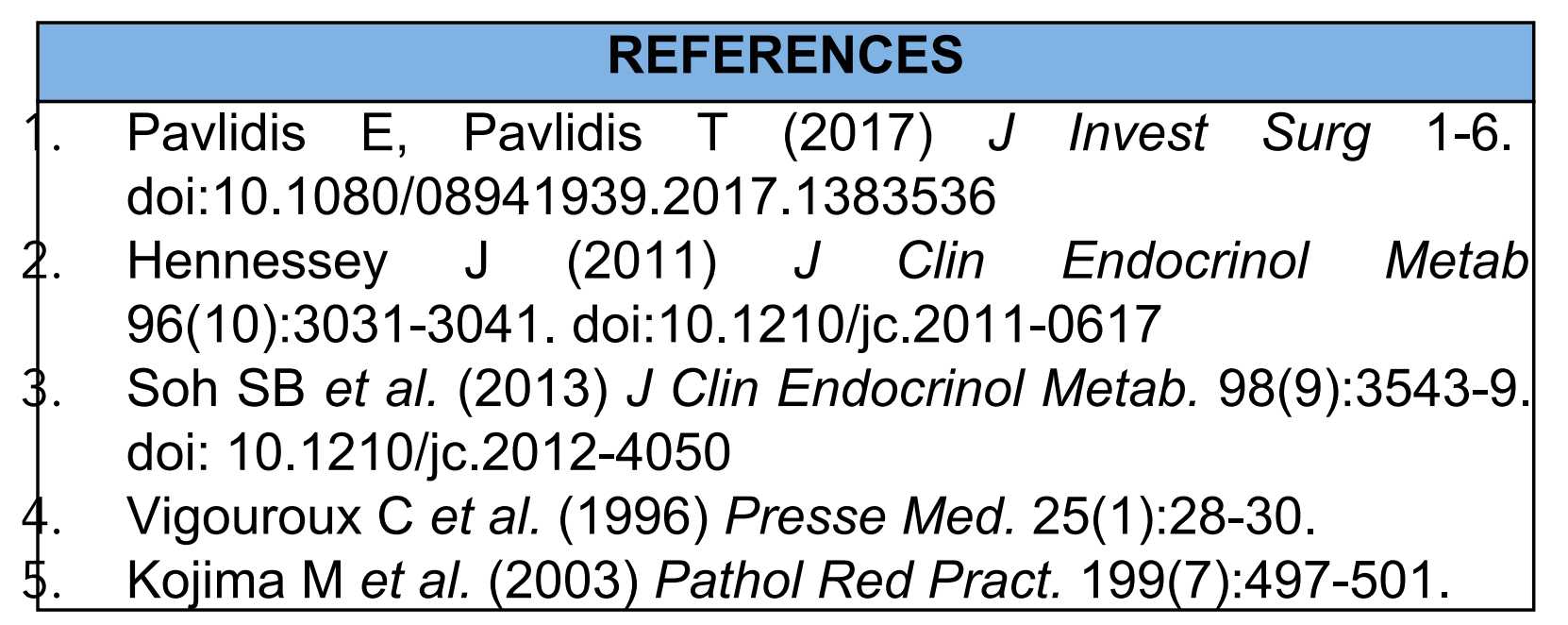

\section{ACKNOWLEDGEMENTS}

Many thanks to the ENT and Haematology departments at St George's University Hospital Trust for their assistance in obtaining case notes and imaging used for the making of this poster. 